\title{
Monte Carlo Estimation of PDM-QPSK/OOK and DQPSK/OOK Hybrid Systems Tolerance Against Nonlinear Effects
}

\author{
Marco Bertolini, Student Member, IEEE, Paolo Serena, Nicola Rossi, and Alberto Bononi
}

\begin{abstract}
We compare through Monte Carlo simulations the performance of polarization-division multiplexed quadrature phase-shift keying (PDM-QPSK)/on-off keying (OOK) and differential QPSK (DQPSK)/OOK hybrid systems, taking into account nonlinear phase noise. DQPSK turns out to be better with optimal dispersion-management, while PDM-QPSK proves to be a good candidate for bit rates higher than $40 \mathrm{~Gb} / \mathrm{s}$.
\end{abstract}

Index Terms-Coherent detection, cross-phase modulation (XPM), optical transmission, phase-shift keying.

\section{INTRODUCTION}

$\mathbf{T}$ HE Internet traffic growth calls for an increased capacity of dense wavelength division multiplexing (DWDM) optical transmission systems. Advanced modulation formats [1] allow us to design optical systems with a bit rate per channel of $40 \mathrm{~Gb} / \mathrm{s}$ or higher and good tolerance against linear and nonlinear impairments. The complete substitution of deployed nonreturn to zero on-off keying (NRZ-OOK) systems is a very expensive option, thus a cost effective solution is to upgrade one or more selected channels, following the market demand. Systems that employ two or more modulation formats and possibly different data rates are commonly referred to as "hybrids".

Many different formats have been proposed as candidates for the channel upgrade. Phase shaped binary transmission (PSBT) and differential quadrature phase-shift keying (DQPSK) have been studied both numerically [2] and experimentally [3], [4] in hybrid systems. To the authors knowledge, polarization-division multiplexing coherent QPSK (PDM-QPSK) has been only studied once experimentally in a hybrid environment [5]. PSBT and DQPSK require a simpler incoherent receiver, but allow for a maximum bit rate of $40 \mathrm{~Gb} / \mathrm{s}$ in a DWDM system (channel spacing $50 \mathrm{GHz}$ ). On the other hand, PDM-QPSK needs a quite complex coherent receiver and a postreception digital signal processing (DSP) unit, but allows for very high bit rates ( 80 or $100 \mathrm{~Gb} / \mathrm{s}$ ) on the same grid.

The purpose of this letter is to compare numerically by means of Monte Carlo simulations the performance of PDM-QPSK and DQPSK in a hybrid scenario and try to determine which

Manuscript received July 18, 2008; revised October 02, 2008. Current version published December 24, 2008. This work was supported by Alcatel-Lucent Bell Laboratories, France.

The authors are with the Dipartimento di Ingegneria dell'Informazione, Università degli Studi di Parma, 43100 Parma, Italy (e-mail: bertolini@ tlc.unipr.it; serena@tlc.unipr.it; rossi_n@tlc.unipr.it; bononi@tlc.unipr.it).

Color versions of one or more of the figures in this letter are available online at http://ieeexplore.ieee.org.

Digital Object Identifier 10.1109/LPT.2008.2008027 one is the most suitable for a system upgrade. We will account for linear and nonlinear system impairments and will highlight pros and cons of the proposed solutions.

\section{Simulation Setup}

All the simulations were performed using an internally developed optical transmission simulator. We tested a five-channel system on a $50-\mathrm{GHz}$ grid that propagates along a dispersion managed $(\mathrm{DM})$ system composed of $20 \times 100 \mathrm{~km}$ spans of Teralight fiber $(\mathrm{D}=8 \mathrm{ps} / \mathrm{nm} / \mathrm{km}, \alpha=0.2 \mathrm{~dB} / \mathrm{km}$, $\gamma=1.7(1 / \mathrm{W} \cdot \mathrm{km})$ at $1550 \mathrm{~nm})$ and single mode fiber (SMF, $\mathrm{D}=16 \mathrm{ps} / \mathrm{nm} / \mathrm{km}, \alpha=0.2 \mathrm{~dB} / \mathrm{km}, \gamma=1.3(1 / \mathrm{W} \cdot \mathrm{km})$ at $1550 \mathrm{~nm})$. All the results refer to the central channel. We separately verified that increasing the number of channels does not cause significant variations of performance [3]. The line was composed of 20 identical spans, each comprising transmission and linear compensating fibers, followed by an amplifier with flat gain and a noise figure of $6 \mathrm{~dB}$. Purely linear pre-/post-compensating fibers were inserted before/after the transmission link.

The even channels were always NRZ-OOK modulated at a bit rate of $10 \mathrm{~Gb} / \mathrm{s}$ (10 Gbaud). The odd channels were in turn $40 \mathrm{~Gb} / \mathrm{s}$ DQPSK (20 Gbaud), $40 \mathrm{~Gb} / \mathrm{s}$ PDM-QPSK (10 Gbaud), or $80 \mathrm{~Gb} / \mathrm{s}$ PDM-QPSK (20 Gbaud). The OOK channels were modulated using pseudo-random binary sequences (PRBS) with different seeds and length $2^{9}$, while the DQPSK and PDMQPSK were modulated using a pseudo-random quaternary sequence (PRQS) of length $4^{5}$. All channels had the same average power and were synchronous at the input of the pre-fiber. We verified that inserting random delays among channels shows very limited impact thanks to the decorrelation induced by precompensating and inline fibers. OOK channels were copolarized with one polarization of coherent channels in PDM-QPSK configuration.

The propagation of signal and amplified spontaneous emission (ASE) noise along the fibers was modeled using a variable step-size split step Fourier method (SSFM), that takes into account all linear and nonlinear effects, except polarization mode dispersion (PMD). The maximum nonlinear phase rotation per step was $3 \cdot 10^{-3}$ rad which we verified to be small enough for the considered system.

The DQPSK channel was received using a second order super-Gaussian optical filter with $3-\mathrm{dB}$ bandwidth $2 \times$ baud rate $(20$ or $40 \mathrm{GHz}$ ), followed by a standard receiver and a fifth-order Bessel filter with 3-dB bandwidth $0.65 \times$ baud rate ( 6.5 or $13 \mathrm{GHz}$ ) [6]. The PDM-QPSK receiver used the same filters and was composed of two coherent mixers (one for each polarization), used to combine the signal with the local 


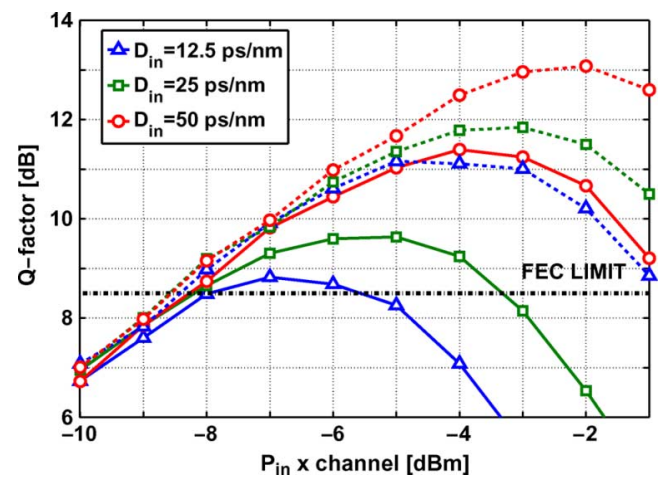

Fig. 1. $Q$-factor versus $P_{\text {in }}$ for DQPSK at $40 \mathrm{~Gb} / \mathrm{s}$ with $10 \mathrm{G}$ OOK neighboring channels. Dashed lines with SMF fiber, solid lines with Teralight.

oscillator, followed by four balanced photodetectors and the DSP unit. The incoming signal is sampled at twice the symbol rate and no quantization is performed. The receiver is the same as described in [7], with no digital dispersion compensation.

\section{RESULTS AND DISCUSSION}

For each configuration, we measured the $Q$-factor as a function of the average launched power $P_{\text {in }}$, for three different values of residual dispersion per span, $D_{\text {in }}=12.5,25$, and $50 \mathrm{ps} / \mathrm{nm} \_$and using Teralight [8] and SMF. The $Q$-factor was computed from Monte Carlo simulations of the bit-error rate (BER) in order to account for nonlinear phase noise, which is often neglected [2]. The simulations were stopped when the relative estimation error on BER reached $20 \%$ with a Gaussian confidence of $95 \%$, providing in each case at least 100 error counts. The precompensating fiber cumulated dispersion was $-292,-411$, and $-649 \mathrm{ps} / \mathrm{nm}$ for the three tested $D_{\text {in }}$, respectively, when using Teralight fiber, and $-488,-607$, and $-844 \mathrm{ps} / \mathrm{nm}$ when using SMF. These values were chosen using the "straight line rule" [9]. The dispersion of the post-compensating fiber was optimized for a QPSK transmission using Karhunen-Loève method [6], assuming white noise. Typically, the residual total dispersion was close to zero and in every tested case it was within the range $[-40 ; 40] \mathrm{ps} / \mathrm{nm}$.

In such systems, the main nonlinear impairment was found to be the cross-phase modulation (XPM) due to $10 \mathrm{G}$ OOK channels on 40G/80G channels [3]. Though not reported here, we ran single channel simulations for DQPSK and $80 \mathrm{G}$ PDM-QPSK configurations with Teralight fiber. We verified that cross-channel effects are dominant. Among these, four-wave mixing is negligible because of the sizable inline dispersion, while switching cross-polarization effects in simulation of PDM-QPSK systems did improve the $Q$-factor by no more than $0.7 \mathrm{~dB}$ over the shown input power range. Hence, we conclude that XPM is by far the dominant nonlinear effect.

Figs. 1-3 sketch the measured $Q$-factor versus launched power for the configurations under investigation. The best performance is always obtained with SMF fiber which is not surprising since increasing the dispersion is known to reduce XPM. Also, the use of SMF reduces the impact of $D_{\text {in }}$ (the $Q$-factor curves for SMF are closer than for Teralight), thus relaxing the dispersion mapping constraints. From a comparison of Fig. 1 and Fig. 2, we note that the best $Q$-factor is better for the DQPSK case $(\sim 0.8 \mathrm{~dB}$ with Teralight and $\sim 1.5 \mathrm{~dB}$ with SMF). The 40G PDM-QPSK shows an enhanced

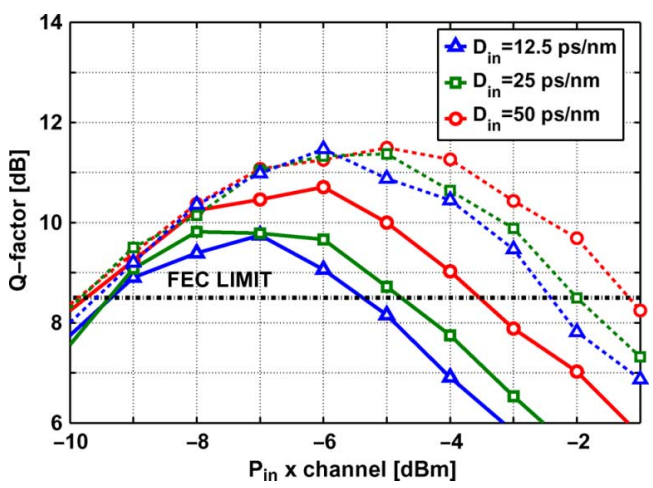

Fig. 2. $Q$-factor versus $P_{\text {in }}$ for PDM-QPSK at $40 \mathrm{~Gb} / \mathrm{s}$ with $10 \mathrm{G}$ OOK neighboring channels. Dashed lines with SMF fiber, solid lines with Teralight.

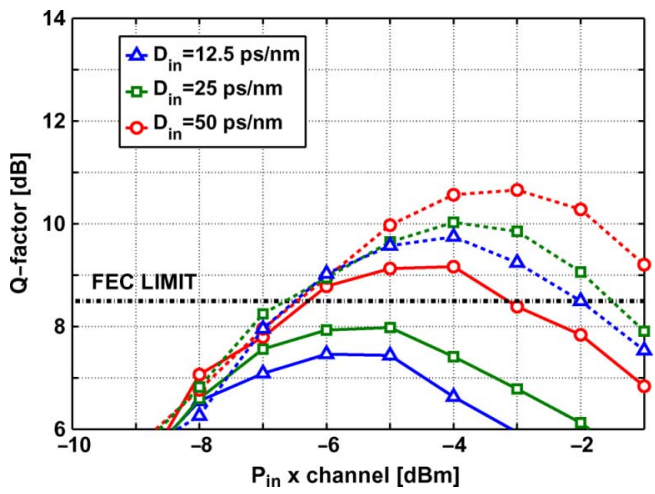

Fig. 3. $Q$-factor versus $P_{\text {in }}$ for PDM-QPSK at $80 \mathrm{~Gb} / \mathrm{s}$ with $10 \mathrm{G}$ OOK neighboring channels. Dashed lines with SMF fiber, solid lines with Teralight.

TABLE I

IMPROVEMENT OF BEST $Q$-FACTOR [in decibels] WHEN USING 100 GHz SPACING ON TERALIGHT Fiber

\begin{tabular}{|c|c|c|c|}
\hline & DQPSK 40G & PDM-QPSK 40G & PDM-QPSK 80G \\
\hline \hline$D_{\text {in }}=12.5 \mathrm{ps} / \mathrm{nm}$ & 1.96 & 0.78 & 0.33 \\
\hline$D_{\text {in }}=25 \mathrm{ps} / \mathrm{nm}$ & 2.24 & 1.17 & 0.24 \\
\hline$D_{\text {in }}=50 \mathrm{ps} / \mathrm{nm}$ & 2.12 & 0.83 & 0.24 \\
\hline
\end{tabular}

optical signal-to-noise ratio (OSNR) sensitivity in the linear region on the left of the maximum $Q$-factor. On the other hand, DQPSK shows a superior nonlinear threshold (NLT, defined as $P_{\text {in }}$ per channel at best $Q$-factor). Fig. 3 shows that $80 \mathrm{G}$ PDM-QPSK suffers from a reduced OSNR sensitivity due to its higher bit rate $\left(\sim 3 \mathrm{~dB}\right.$ in $Q$-factor at $\left.P_{\text {in }}=-8 \mathrm{dBm}\right)$ and for low values of $D_{\text {in }}$ the nonlinear penalties are enhanced, compared to 40G PDM-QPSK. However, the NLT is better than 40G PDM-QPSK ( $2 \mathrm{~dB}$ with both fibers). Over such a long distance this configuration cannot offer adequate performance. However, with a careful DM (higher values of $D_{\text {in }}$ ) and on shorter links $(1000 \div 1500 \mathrm{~km})$, PDM-QPSK could be a good candidate for $80 \mathrm{G}$ or even $100 \mathrm{G}$ channel upgrades.

We also investigated the effect of increasing the channel spacing from 50 to $100 \mathrm{GHz}$ on a Teralight fiber. Table I reports the improvement of the best $Q$-factor for the considered configurations. As expected, the $Q$-factor is always better at $100 \mathrm{GHz}$, but this effect is more evident for DQPSK ( $2 \mathrm{~dB})$ than for 40/80G PDM-QPSK ( $\sim 1$ and $\sim 0.3 \mathrm{~dB}$, respectively). This is probably due to the fact that increasing the channel spacing does not reduce nonlinear depolarization [10]. 


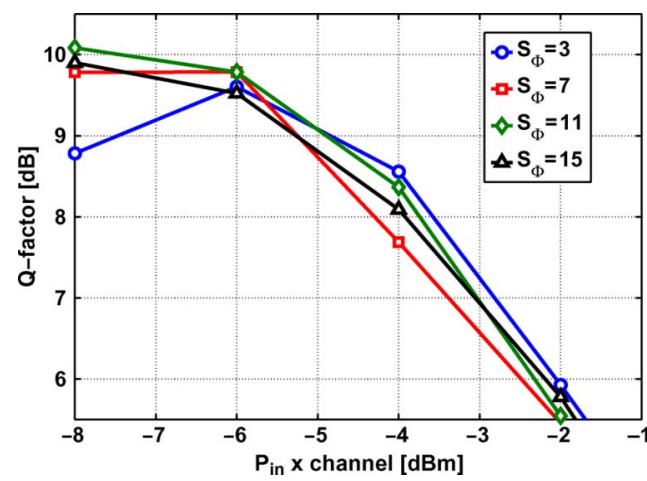

Fig. 4. $Q$-factor versus $P_{\text {in }}$ of PDM-QPSK $40 \mathrm{~GB} / \mathrm{s}+\mathrm{OOK}, D_{\text {in }}=25 \mathrm{ps} / \mathrm{nm}$ on Teralight fiber for four different values of $S_{\Phi}$.

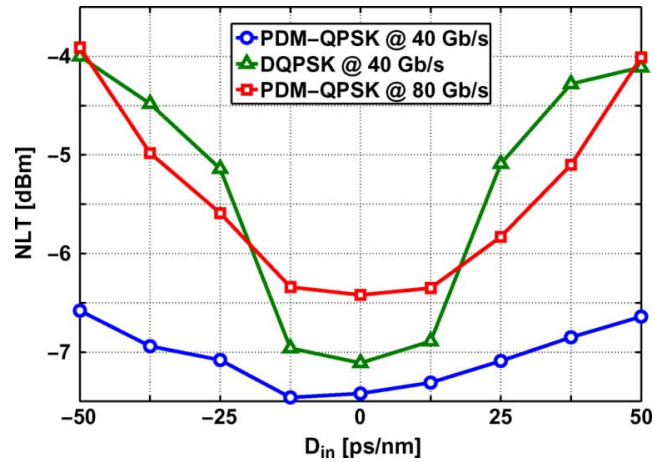

Fig. 5. NLT versus $D_{\text {in }}$ of the three examined configurations on Teralight fiber.

A possible solution to improve PDM-QPSK robustness against SPM and XPM is to improve the phase estimation algorithm. In our simulations, we used a Viterbi\&Viterbi [7]. The number of samples used to average the phase $\left(S_{\Phi}\right)$ was seven. It is a well-known fact that the value of $S_{\Phi}$ has an influence on the quality of the estimation process; high values of $S_{\Phi}$ are better in ASE-limited systems, while smaller values are better when nonlinear effects arise. Fig. 4 shows the performance of the 40G PDM-QPSK+OOK system, using $D_{\text {in }}=25 \mathrm{ps} / \mathrm{nm}$ and Teralight fiber, for different values of $S_{\Phi} . S_{\Phi}=7$ is near the optimum for low values of $P_{\text {in }}$, but gives a penalty of almost $1 \mathrm{~dB}$ for $P_{\text {in }}=-4 \mathrm{dBm}$. Using smaller values of $S_{\Phi}$ (es. 3) could thus reduce the impact of XPM, but could be insufficient to provide a decisive improvement. On the other hand, the DSP unit allows for a suboptimal DM design. Comparing Figs. 1 and 2 for $D_{\text {in }}=12.5 \mathrm{ps} / \mathrm{nm}$ with Teralight fiber, we see that the $Q$-factor of PDM-QPSK is decreased by $2 \mathrm{~dB}$ w.r.t. $D_{\text {in }}=50 \mathrm{ps} / \mathrm{nm}$, while the $Q$-factor of DQPSK is $4.5 \mathrm{~dB}$ smaller. The enhanced tolerance of PDM-QPSK is due to the constant modulus algorithm (CMA) used at the receiver that acts as a generic adaptive equalizer, thus relaxing the impact of DM [7].

Finally, Fig. 5 shows the NLT as a function of $D_{\text {in }}$ using Teralight fiber. These curves are obtained by interpolating the available values of $Q$-factor versus $P_{\mathrm{in}}$. The NLT always grows for increasing values of $\left|D_{\text {in }}\right|$, because residual inline dispersion reduces the dominant XPM impairment. The NLT of $40 \mathrm{G}$
PDM-QPSK is the lowest but its value is little dependent on $D_{\text {in }}(1 \mathrm{~dB})$. On the other hand, the NLT for 40G DQPSK and $80 \mathrm{G}$ PDM-QPSK is higher, but it decreases by more than 3 and $2.5 \mathrm{dBm}$, respectively, at $D_{\text {in }}=0$.

\section{CONCLUSION}

We provided a numerical Monte Carlo comparison between 40G DQPSK, 40G PDM-QPSK, and 80G PDM-QPSK, three of the best candidates for the deployment of hybrid systems, assessing their tolerances against non-linear effects.

PDM-QPSK is a very promising format that will enable us to deliver bit rates up to $100 \mathrm{~Gb} / \mathrm{s}$ per channel. However, in order to ensure enhanced tolerance against cross-channel nonlinear impairments and thus extend the reach, the phase should be carefully estimated. At $40 \mathrm{~Gb} / \mathrm{s}$, PDM-QPSK is strongly affected by XPM while, with proper DM design, DQPSK has proven to be the best choice among the considered formats for this bit rate in ultra-long-haul systems.

\section{ACKNOWLEDGMENT}

The authors would like to thank G. Charlet of Alcatel-Lucent Bell Laboratories, France, for fruitful discussions.

\section{REFERENCES}

[1] P. J. Winzer and R.-J. Essiambre, “Advanced modulation formats for high-capacity optical transport networks," J. Lightw. Technol., vol. 24, no. 12 , pp. 4711-4728, Dec. 2006.

[2] M. Lefrancois, F. Houndonougbo, T. Fauconnier, G. Charlet, and S. Bigo, "Cross comparison of the nonlinear impairments caused by 10 Gbit/s neighboring channels on a $40 \mathrm{Gbit} / \mathrm{s}$ channel modulated with various formats, and over various fiber types," in Proc. OFC, Anaheim, CA, 2007, Paper JThA44.

[3] S. Chandrasekhar and X. Liu, "Impact of channel plan and dispersion map on hybrid DWDM transmission of 42.7-Gb/s DQPSK and 10.7-Gb/s OOK on 50-GHz grid," IEEE Photon. Technol. Lett., vol. 19, no. 22, pp. 1801-1803, Nov. 15, 2007.

[4] M. Birk, C. Skolnick, B. Curto, R. Marlieb, T. Schmidt, and R. Saunders, "Field trial of a $40 \mathrm{Gbit} / \mathrm{s}$ PSBT channel upgrade to an installed 1700 km 10 Gbit/s system," in Proc. OFC, Anaheim, CA, 2005, Paper OTuH3.

[5] O. Bertran Pardo, J. Renaudier, H. Mardoyan, P. Tran, G. Charlet, and $\mathrm{S}$. Bigo, "Investigation of design options for overlaying $40 \mathrm{~Gb} / \mathrm{s}$ coherent PDM-QPSK channels over a $10 \mathrm{~Gb} / \mathrm{s}$ system infrastructure," in Proc. OFC, San Diego, CA, 2008, Paper OTuM5.

[6] P. Serena, A. Orlandini, and A. Bononi, "Parametric-gain approach to the analysis of single-channel DPSK/DQPSK systems with nonlinear phase noise," J. Lightw. Technol., vol. 24, no. 5, pp. 2026-2037, May 2006.

[7] J. Renaudier, G. Charlet, M. Salsi, O. Bertrand Pardo, H. Mardoyan, P. Tran, and S. Bigo, "Linear fiber impairments mitigation of 40-Gbit/s polarization-multiplexed QPSK by digital processing in a coherent receiver," J. Lightw. Technol., vol. 26, no. 1, pp. 36-42, Jan. 2008.

[8] M. Bertolini, P. Serena, N. Rossi, and A. Bononi, "Numerical Monte Carlo comparison between coherent PDM-QPSK/OOK and incoherent DQPSK/OOK hybrid systems," in Proc. ECOC, Bruxelles, Belgium, 2008, Paper P.4.15.

[9] Y. Frignac, J.-C. Antona, and S. Bigo, "Enhanced analytical engineering rule for fast optimization dispersion maps in $40 \mathrm{Gbit} / \mathrm{s}$-based transmission," in Proc. OFC, San Diego, CA, 2008, Paper OTuN3.

[10] S. Bigo, G. Charlet, O. Bertrand Pardo, and J. Renaudier, "Characterization of the impact of non-linear effects in coherent transmission experiments," in Dig. IEEE/LEOS Summer Topical Meetings 2008, Jul. 2008, pp. 125-126. 